\title{
Transcript analysis of the extended hyp-operon in the cyanobacteria Nostoc sp. strain PCC 7120 and Nostoc punctiforme ATCC 29133
}

\author{
Marie Holmqvist, Pia Lindberg ${ }^{\dagger}$, Åsa Agervald ${ }^{\dagger}$, Karin Stensjö and Peter Lindblad ${ }^{*}$
}

\begin{abstract}
Background: Cyanobacteria harbor two [NiFe]-type hydrogenases consisting of a large and a small subunit, the Hup- and Hox-hydrogenase, respectively. Insertion of ligands and correct folding of nickel-iron hydrogenases require assistance of accessory maturation proteins (encoded by the hyp-genes). The intergenic region between the structural genes encoding the uptake hydrogenase (hupSL) and the accessory maturation proteins (hyp genes) in the cyanobacteria Nostoc PCC 7120 and N. punctiforme were analysed using molecular methods.

Findings: The five ORFs, located in between the uptake hydrogenase structural genes and the hyp-genes, can form a transcript with the hyp-genes. An identical genomic localization of these ORFs are found in other filamentous, $\mathrm{N}_{2}$-fixing cyanobacterial strains. In N. punctiforme and Nostoc PCC 7120 the ORFs upstream of the hypgenes showed similar transcript level profiles as hups (hydrogenase structural gene), nifD (nitrogenase structural gene), hypC and hypF (accessory hydrogenase maturation genes) after nitrogen depletion. In silico analyzes showed that these ORFs in N. punctiforme harbor the same conserved regions as their homologues in Nostoc PCC 7120 and that they, like their homologues in Nostoc PCC 7120, can be transcribed together with the hyp-genes forming a larger extended hyp-operon. DNA binding studies showed interactions of the transcriptional regulators CalA and CalB to the promoter regions of the extended hyp-operon in N. punctiforme and Nostoc PCC 7120.

Conclusions: The five ORFs upstream of the hyp-genes in several filamentous $\mathrm{N}_{2}$-fixing cyanobacteria have an identical genomic localization, in between the genes encoding the uptake hydrogenase and the maturation protein genes. In N. punctiforme and Nostoc PCC 7120 they are transcribed as one operon and may form transcripts together with the hyp-genes. The expression pattern of the five ORFs within the extended hyp-operon in both Nostoc punctiforme and Nostoc PCC 7120 is similar to the expression patterns of hupS, nifD, hypF and hypC. CalA, a known transcription factor, interacts with the promoter region between hupSL and the five ORFs in the extended hyp-operon in both Nostoc strains.
\end{abstract}

\section{Background}

Cyanobacteria are an ancient group of organisms capable of both oxygenic photosynthesis and hydrogen evolution. Molecular hydrogen $\left(\mathrm{H}_{2}\right)$ is produced by nitrogenases as a by-product when fixing atmospheric dinitrogen $\left(\mathrm{N}_{2}\right)$ while uptake hydrogenases recapture the molecular hydrogen and oxidize it, to prevent energy losses from the cells. In addition, if present bidirectional hydrogenases have the capacity to both produce and

\footnotetext{
* Correspondence: Peter.Lindblad@fotomol.uu.se

+ Contributed equally

Department of Photochemistry and Molecular Science, The Ångström Laboratories, Uppsala University, Box 523, SE-751 20 Uppsala, Sweden
}

oxidize $\mathrm{H}_{2}$ [1-3]. Hydrogenases and nitrogenases are oxygen sensitive enzymes $[1,2]$ and therefore cyanobacteria have developed different strategies to maintain enzyme activity by separating the enzymes from oxygenic photosynthesis either spatially, e.g. in heterocysts $[2,4,5]$, or temporally [5]. In heterocystous cyanobacteria nitrogenase and uptake hydrogenase are expressed in heterocysts, while the bidirectional hydrogenase is present in both heterocysts and vegetative cells. Neither the uptake nor the bidirectional hydrogenases are universally present among cyanobacteria. However, all filamentous strains examined so far capable of $\mathrm{N}_{2}$-fixation contain an uptake hydrogenase $[2,3,6]$. 
All cyanobacterial hydrogenases belong to the NiFe class of hydrogenases [3,6]. The uptake hydrogenase consists of a small and a large subunit, encoded by hupS and $h u p L$, respectively $[1,2,5]$. The large subunit, HupL, harbors the $\mathrm{Ni}$ and Fe containing active site $[3,6]$. The assembly of the active site and correct folding of HupL is a complex process, requiring assistance of accessory proteins, encoded by at least hypABCDEF, and a hydrogenase specific protease, encoded by hup $W[3,7]$. The hyp-genes (hyp for hydrogenase pleiotropic) and the Hyp-proteins have been studied mainly in Escherichia coli [7]. However, mutational analyses in e.g. Synechocystis sp. PCC 6803 have shown that the corresponding cyanobacterial hyp-genes most likely have similar function $[6,8]$.

The small subunit, HupS, mediates electron transport from the active site located in the large subunit to redox partners and downstream reactions through a set of FeS clusters [3,7]. Little is known about the cyanobacterial maturation process and assembly of FeS clusters in the small subunit. Three different kinds of FeS cluster biosynthesis systems have been identified in bacteria, the ISC (iron-sulphur cluster), SUF (mobilization of sulfur) and NIF (nitrogen fixation) system. They all have in common that they require a FeS cluster scaffolding protein (IscU and IscA, SufA, NifU) and a cysteine desulfurase (IscS, SufS, NifS), providing elemental sulphur $[3,9]$. Cyanobacteria lack homologues to iscU but the gene $n f u$, encoding a protein with high similarity to the C-terminal domain of $\mathrm{NifU}$, is present. In cyanobacteria SufA and IscA seem to have a more regulatory function, e.g. sensing of redox stress and being involved in FeS cluster assembly under iron homeostasis. Nfu has been suggested to be involved in general FeS cluster assembly and is considered to be an essential FeS cluster scaffold protein [10].

There have been reports of proteins participating in the maturation process of the small subunit of uptake hydrogenases. The legume endosymbiont Rhizobium leguminosarum bv. viciae strain UPM791 harbors a subcluster of five genes, hupGHIJK, which has been connected to the maturation of the small subunit of the uptake hydrogenase. The subcluster is part of the hydrogenase gene cluster due to its location between the structural genes and the hyp-genes, and is preceded by a promoter $\left(\mathrm{P}_{3}\right)$ upstream of hup $G$ [11-13]. Homologues can be found in other aerobic, NiFe-type uptake hydrogenase containing bacteria such as Azotobacter vinelandii, Rhodobacter capsulatus, Ralstonia eutropha H16, Azotobacter chroococcum and Bradyrhizobium japonicum [14-16]. The presence of HupGHIJ appears to be connected to oxygen dependent microorganisms, since no homologues have been found in strictly anaerobic bacteria $[11,16]$. HupH forms a direct complex with the small subunit precursor, pre-HupS [11]. In addition, an interaction between the uptake hydrogenase small subunit Tat signal peptide and the HupH and HupG homologues, HoxQ and HoxO, has been shown in Ralstonia eutropha H16 [14,17,18]. The proposed functions of HupGHIJ in R. leguminosarum and HoxOQ in R. eutropha are to protect the FeS clusters in the small subunit from oxygen, stabilizing the small subunit complexes and preventing premature translocation of the small subunit to the periplasm by masking the Tat signal peptide $[11,18]$.

Recently, we presented a set of putative additional hyp-genes in the cyanobacterium Nostoc sp. strain PCC 7120 (also called Anabaena sp. strain PCC 7120) [19]. These five ORFs, asr0689 to alr0693, are located in between hupSL and the hyp-genes (Figure 1, Table 1). The same genomic organization can be found in other $\mathrm{N}_{2}$-fixing strains such as the filamentous, heterocyst forming strains Nostoc punctiforme ATCC 29133, Nodularia spumigena CCY9414 and Anabaena variabilis ATCC 29413 as well as in the filamentous, non-heterocyst forming strains Lyngbya majuscula CCAP 1446/4 [19]. This genomic localization is identical to the organization of hupGHIJK discussed above. The conservation of these ORFs located upstream of the hyp-genes in $\mathrm{N}_{2}$-fixing cyanobacteria suggest that they may serve an important role in hydrogen metabolism, putatively in the maturation of the small subunit of the uptake hydrogenase. In Nostoc PCC 7120 the ORFs upstream of the hyp-genes can form a transcript together with the hyp-genes and a putative transcription start point (tsp) was identified upstream of asr0689 [19]. The ORFs upstream of the hyp-genes have been annotated as encoding unknown proteins. However, the deduced amino acid sequences harbor conserved regions such as tetratricopeptide repeats (TPR), NHL repeats (NHL from NCL-1, HT2A and Lin-41) and a NifU like domain [19]. TPR domains are known to mediate protein-protein interactions [20-22], and NHL repeats may also have a similar function [23].

N. punctiforme and Nostoc PCC 7120 are two filamentous, heterocyst forming cyanobacterial strains used in the present study. Both possess an uptake hydrogenase while Nostoc PCC 7120 in addition possesses a bidirectional enzyme $[2,24,25]$. This makes the two strains interesting to compare due to the presence of only one set of hyp-genes in both genomes [19,26,27]. In this study we have further investigated the ORFs upstream of the hyp-genes by examining their transcript level pattern after nitrogen depletion in N. punctiforme and Nostoc PCC 7120. The results were compared to the transcript level pattern for genes known to be involved in hydrogen metabolism, such as hupS (encoding the 


\section{A Npun_R0364 \\ Npun $\bar{R} 0365$ \\ Npun_R0366}

hupL hupS Npun R0367

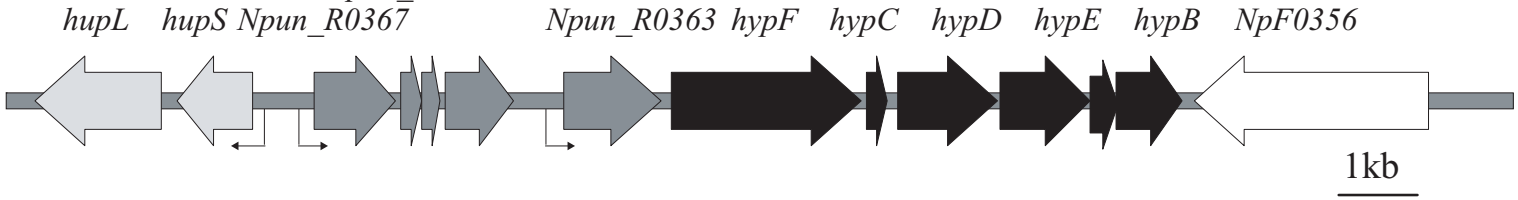

B

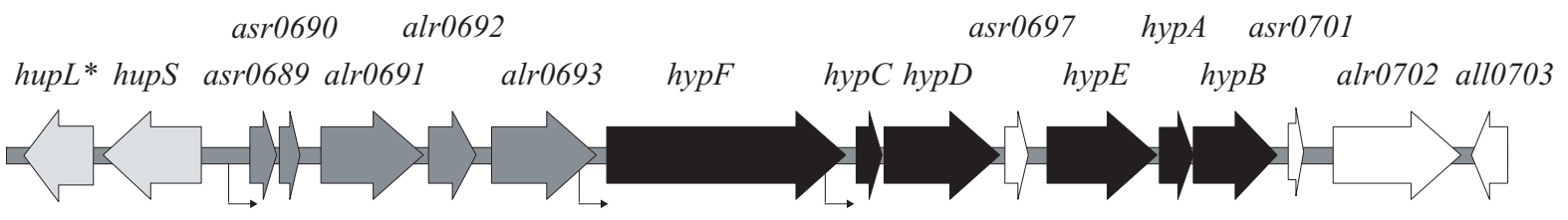

$1 \mathrm{~kb}$

Figure 1 Genomic arrangement of the ORFs upstream of the hyp-genes in Nostoc punctiforme ATCC 29133 and Nostoc sp. strain PCC 7120. (A) In the filamentous, heterocyst forming cyanobacterial strain N. punctiforme the five ORFs upstream of the hyp-genes are located upstream of the uptake hydrogenase structural genes, hupSL, and in between hupSL and the hyp-genes, hypFCDEAB. (B) The same genomic arrangement can be found in the filamentous, heterocyst forming cyanobacterial strain Nostoc PCC 7120. This genomic arrangement of the ORFs upstream of the hyp-genes seems to be conserved in filamentous cyanobacteria harboring an uptake hydrogenase [19] * indicates the $5^{\prime}$ end of hupL (encoding the N-terminal end of HupL) as it is annotated in vegetative cells. The identified tsps upstream of hupSL [36], Npun_R0363 [27] and Npun_R0367 (this work) in ATCC 29133 and upstream of asr0689, hypF and hypC in Nostoc PCC 7120 [19] are indicated by arrows.

uptake hydrogenase small subunit), nifD (encoding a nitrogenase subunit) and hypC and hypF (encoding Hyp-proteins). We also tested if the ORFs upstream of the hyp-genes in N. punctiforme may be, like in Nostoc PCC 7120, transcribed together with the hyp-operon. The ORFs upstream of the hyp-genes in N. punctiforme were analyzed in silico and the region examined experimentally for transcription start points. In addition, different DNA affinity studies were used to analyze binding of transcriptional regulators to the promoter region of the extended hyp-operon in both $N$. punctiforme and Nostoc PCC 7120.

\section{Methods}

\section{Strains and culture conditions}

The filamentous heterocystous cyanobacterial strains Nostoc punctiforme ATCC 29133 and Nostoc sp. strain PCC 7120 (also named Anabaena sp. strain PCC 7120) were cultured in $B G 11_{0}$ medium [28], sparged with air and grown at $25^{\circ} \mathrm{C}$, at a continuous irradiance of 40 $\mu \mathrm{mol}$ of photons $\mathrm{m}^{-2} \mathrm{~s}^{-1}$ [29]. For non $\mathrm{N}_{2}$-fixing conditions the $\mathrm{BG} 11_{0}$ medium was supplemented with $5 \mathrm{mM}$ $\mathrm{NH}_{4} \mathrm{Cl}$ and $5 \mathrm{mM}$ MOPS (pH 7.8).

\section{In silico genome analyses of the ORFs upstream of the} hyp-genes

Sequence homology search for conserved domains in the ORFs upstream of the hyp-genes of $N$. punctiforme was performed in Cyanobase (http://genome.kazusa.or. jp/cyanobase/), UniProt Knowledgebase (Swiss-Prot and TrEMBL at http://www.expasy.org/sprot/) and Pfam (http://pfam.sanger.ac.uk). The deduced protein sequences of HupGHIJ were blasted against the N. punctiforme and Nostoc PCC 7120 and protein database at Cyanobase (http://genome.kazusa.or.jp/cyanobase) and National Center for Biotechnology Information (NCBI) (http://www.ncbi.nlm.nih.gov/).

\section{Heterocyst preparation}

Heterocysts from Nostoc PCC 7120 were isolated as previously described with a few modifications [30,31]. The incubation step with lysozyme was prolonged to 2 hours and the sonication step decreased to 3 intervals of $10 \mathrm{~s}$. The pellet, containing heterocysts, were washed 4 times in extraction buffer and centrifugated at $4^{\circ} \mathrm{C}$ at, in order, $1000 \times \mathrm{g}, 1000 \times \mathrm{g}, 750 \times \mathrm{g}, 500 \times \mathrm{g}$ and $250 \times \mathrm{g}$ for 5 min respectively.

Table 1 The ORFs upstream of the hyp-genes in Nostoc sp. strain PCC 7120 and their homologues in Nostoc punctiforme ATCC 29133

\begin{tabular}{llllll}
\hline Nostoc sp. strain PCC 7120 & asr0689 & asr0690 & alr0691 & alr0692 & alr069 \\
\hline Nostoc punctiforme ATCC 29133 & Npun_R0366 & Npun_R0365 & Npun_R0367 & Npun_R0364 & Npun_R0363 \\
\hline
\end{tabular}


Nucleic acid isolation and analysis

Genomic DNA and RNA were isolated from N. punctiforme and Nostoc PCC 7120 cultures as previously described $[19,29]$. The rRNA quality was analyzed with the Experion System (Bio-Rad Laboratories) according to the manufacturer's instructions. The concentration was determined by absorbance measurements using Cary Win UV (Varian). Prior to RT-reactions RNA was treated with DNaseI (Fermentas) according to the instructions of the manufacturer.

\section{Primer construction}

All oligonucleotides used are listed in Tables 2 and 3. Primers were designed either by Primer3 program (http://frodo.wi.mit.edu/primer3/) or manually. The secondary structure was analyzed with the Primer design utility program EazyPrimer ${ }^{\mathrm{TM}}$ (http://www.cybergene.se/ EazyPrimer.htm) and the primers blasted against their corresponding N. punctiforme or Nostoc PCC 7120 genome at Cyanobase (http://genome.kazusa.or.jp/cyanobase/), to check their specificity.

\section{Transcript analysis}

Reverse transcription (RT) reactions were performed according to the manufacturer's instructions with either the iScript $^{\mathrm{TM}}$ cDNA Synthesis Kit (Bio-Rad Laboratories) containing random primers, using $0.25 \mu \mathrm{g}$ total RNA from $N$. punctiforme and $0.5 \mu \mathrm{g}$ total RNA from Nostoc PCC 7120, or the RevertAid ${ }^{\text {TM }}$ First Strand cDNA Synthesis Kit (Fermentas) and gene specific primers, using $0.5 \mu \mathrm{g}$ total RNA from $N$. punctiforme. The cultures were grown under either $\mathrm{N}_{2}$-fixing or non $\mathrm{N}_{2}$-fixing conditions. PCR amplifications using cDNAs of the respective genes were performed using corresponding primers (Table 2). To ensure that the PCR reaction of the reference gene, $23 S$, were not saturated a semi-quantitative analysis of $23 S$ PCR product from $N$. punctiforme and Nostoc PCC 7120 were done by removing a sample every $2^{\text {nd }}$ cycle from, cycle 8 to 26 , and comparing the amount of transcript (data not shown). PCR were used to amplify the regions between hupS to Npun_R0367 (using primers hupS forward and Npun_R0367 reverse), Npun_R0367 to Npun_R0366 (using primers Npun_R0367 forward and Npun_R0366 reverse), Npun_R0366 to Npun_R0365 (using primers Npun_R0366 forward and Npun_R0365 reverse), Npun_R0365 to Npun_R0364 (using primers Npun_R0365 forward and Npun_R0364 reverse), Npun_R0364 to Npun_R0363 (using primers Npun_R0364 forward and Npun_R0363 reverse) and Npun_R0363 to hypF (using primers Npun_R0363 forward and hypF reverse). Negative controls for the RTreaction were RT-PCR on DNaseI treated RNA without
Table 2 Primer oligonucleotides designed and used to generate or amplify cDNA from cyanobacterial cell cultures after nitrogen depletion

\begin{tabular}{|c|c|c|}
\hline Primers & Sequence $5^{\prime}-3^{\prime}$ & Product size \\
\hline \multicolumn{3}{|c|}{ N. punctiforme primers } \\
\hline hups forward & ATTATGGCTACAAGGTGGTG & $215 \mathrm{bp}$ \\
\hline hups reverse & CAACACTGCCTTCAAATACC & \\
\hline nifD forward & CCCAATGTGAAGATGAACCT & $201 \mathrm{bp}$ \\
\hline nifD reverse & GCTGATACTTGGCGATAACT & \\
\hline rnpb forward & AAGCAATAGCAACCATACAGA & $198 \mathrm{bp}$ \\
\hline rnpb reverse & AATTGATCTGGCGGTATCTT & \\
\hline 235 forward & GAAACAGCCCAGACCACC & $197 \mathrm{bp}$ \\
\hline 235 reverse & AGTGAGCTATTACGCACTC & \\
\hline hypC forward & AATCCCCGGACAAATTATAG & $128 \mathrm{bp}$ \\
\hline hypC reverse & ATGTTTCTGCTGCTTCTTGT & \\
\hline hypF forward & TTAAAACAGAAATTGTCCCT & $216 \mathrm{bp}$ \\
\hline hypF reverse & CAACATCGTGGTATTCCTIT & \\
\hline Npun_0363 forward & TATCATCAAGGACGGCTATT & $197 \mathrm{bp}$ \\
\hline Npun_0363 reverse & TCTCCCCAAAAAGCGATGTC & \\
\hline Npun_0364 forward & AACGGTGTGTAGCAGTAGG & $179 \mathrm{bp}$ \\
\hline Npun_0364 reverse & TGGTGGTITTGGTGGTTTG & \\
\hline Npun_0365 forward & GGTTATCTGCCAAGTTATT & $190 \mathrm{bp}$ \\
\hline Npun_0365 reverse & TTGAAGAGAAACGGCATAAT & \\
\hline Npun_0366 forward & TTAACAACGCCAAATCTGAT & $220 \mathrm{bp}$ \\
\hline Npun_0366 reverse & CTAAGAAGATGGCAGGAATT & \\
\hline Npun_0367 forward & AGATAGCACAGGGTTTCAA & $219 \mathrm{bp}$ \\
\hline Npun_0367 reverse & AAATCGGTAATGCCTCTTCA & \\
\hline \multicolumn{3}{|c|}{ Nostoc PCC 7120 primers } \\
\hline hups forward & TTGTTCAGGCAACACCATGT & $154 \mathrm{bp}$ \\
\hline hups reverse & GCCTAAGATGCAATCCCAAA & \\
\hline 235 forward & GCTAAGCGATGTACCGAAGC & $199 \mathrm{bp}$ \\
\hline 235 reverse & TAACCCAGAGTGGACGAACC & \\
\hline hypC forward & GGAATCCCCGGACAAATTAC & $200 \mathrm{bp}$ \\
\hline hypC reverse & GTTCGGCTGCTTCTTGTTC & \\
\hline hypF forward & GTGTCCCGAATGTGAGGACT & $239 \mathrm{bp}$ \\
\hline hypF reverse & GCGTTGCATCACAAGCTAAA & \\
\hline asr0389 forward & ATTTCTGATCAATATGGTCA & $202 \mathrm{bp}$ \\
\hline asr0389 reverse & CCCAGACCCAAAACAATAGC & \\
\hline asr0390 forward & CGGCGAGGTTATTITTGAAG & $196 \mathrm{bp}$ \\
\hline asr0390 reverse & TCTTTGAGAAGAAATGGCATGA & \\
\hline alr0391 forward & TTGGCGAGGATAACCGATAG & $218 \mathrm{bp}$ \\
\hline alr0391 reverse & CTGGGGTGGTCAATCAAGTT & \\
\hline alr0392 forward & CAAGCTGCTTTGGAAGAGGT & $203 \mathrm{bp}$ \\
\hline alr0392 reverse & ACCGCAATCACCGTCACAAT & \\
\hline alr0393 forward & GGGACAGAATGCTAAGGGTA & $200 \mathrm{bp}$ \\
\hline alr0393 reverse & CCCGATTTGGTTCATTCTCC & \\
\hline alr0394 forward & CTTTGTTACAGAAGGGTGAA & $213 \mathrm{bp}$ \\
\hline alr0394 reverse & CAGCAGGACTAACTAATAAC & \\
\hline
\end{tabular}


Table 2 Primer oligonucleotides designed and used to generate or amplify cDNA from cyanobacterial cell cultures after nitrogen depletion (Continued)

\begin{tabular}{lll}
\hline RT primers $\boldsymbol{N}$. punctiforme \\
\hline gsphypF & TCGGATTTCTTGACGAAGG - \\
\hline gspNpun_R0363 & TCGATTATTCCAGAATCAGC & - \\
\hline gspNpun_R0364 & ATCAGGCACGGTTAAGCATT -
\end{tabular}

Primers to amplify CDNA, generated with random primers, from cultures and isolated heterocysts of Nostoc punctiforme ATCC 29133 and cultures of Nostoc sp. strain PCC 7120 are shown as well as gene specific primers for generating CDNA from cultures of Nostoc punctiforme ATCC. Sizes of the resulting PCR product are shown in base pairs (bp). cDNA with gene specific primers were generated through reverse transcriptase reactions (RT).

RT-enzyme. Negative controls for the PCR reactions included PCR amplification without cDNA added and positive controls were made with genomic DNA from N. punctiforme and Nostoc PCC 7120 using the corresponding forward and reverse primer.

\section{PCR and agarose gel electrophoresis}

PCR amplifications were carried out using the enhanced thermostable Taq DNA Polymerase DreamTaq ${ }^{\mathrm{TM}}$ (Fermentas), according to manufacturer's protocol, in a UnoCycler Thermal Cycler (VWR) according to the guidelines provided by the suppliers. For the transcript level analysis an annealing temperature of $60^{\circ} \mathrm{C}$ and 0.8 $\mu \mathrm{l}$ or $0.4 \mu \mathrm{l} \mathrm{cDNA}$ were used (N. punctiforme and Nostoc PCC 7120 respectively) in $10 \mu \mathrm{l}$ reactions. The amount of cycles varied from 15 to 30 cycles for the different samples to avoid saturation of the PCR product in the reactions. The products were visualized on $1 \%$ agarose gels containing thiazole-orange. The gels were run with $1 \mathrm{x}$ sodium boric acid buffer at $160 \mathrm{~V}$ for 15 minutes.
Identification of transcription start points

Transcription start points were located with the 5'RACE System for Rapid Amplification of cDNA Ends, Version 2.0 (Invitrogen), according to the manufacturer's instructions using $1 \mu \mathrm{g}$ total RNA and gene specific primers (Table 3). The resulting PCR products were gel purified using the NucleoSpin ${ }^{\circledR}$ Extract II (MachereyNagel) according to protocol and cloned into the $\mathrm{pCR}^{\circledR}$ 2.1-TOPO ${ }^{\circledR}$ vector (Invitrogen), following to the manufacturer's instructions, before being sequenced at Macrogen Inc. The sequences were subsequently analyzed with BioEdit Sequence Alignment Editor 7.0.5.3. Negative controls included RT-PCR on DNaseI treated RNA without RT-enzyme, dC-tailing without TdT enzyme and PCR amplification without cDNA. Positive controls were made with genomic DNA from N. punctiforme using the corresponding forward and reverse primer.

\section{Protein extraction, DNA affinity assays and mass spectrometry}

Cells from N. punctiforme and Nostoc PCC 7120 cultures were harvested by centrifugation at $3300 \times \mathrm{g}$ for 10 minutes at $4{ }^{\circ} \mathrm{C}$ and washed once in protein buffer (100 mM Tris- $\mathrm{HCl}$ pH 7.5, 1 mM EDTA, 2 mM DTT, $0.5 \%$ Triton X-100, $10 \%$ glycerol and $1 \mathrm{mM}$ PMSF). Proteins were extracted using $0.2 \mathrm{~g}$ of 0.6 - $\mathrm{mm}$-diameter glass beads and a Precellys ${ }^{\circledR} 24$ homogenizer (Bertin Technologies) at a speed of 5500 for $6 \times 30 \mathrm{~s}$ keeping samples on ice between runs. After centrifugation at $10000 \times \mathrm{g}$ for 10 minutes at $4^{\circ} \mathrm{C}$, the supernatants were transferred to new tubes and subjected to further separation by centrifugation at $30000 \times \mathrm{g}$ for 20 minutes at $4^{\circ} \mathrm{C}$. The supernatant was once more transferred to new tubes and the protein concentration was

Table 3 Primer oligonucleotides designed and used to locate novel transcription start points in Nostoc punctiforme ATCC 29133 and to amplify DNA probes for DNA affinity assay and electrophoretic mobility shift assay (EMSA) from N. punctiforme and Nostoc sp. strain PCC 7120 genomic DNA

\begin{tabular}{llll}
\hline Primers & Sequence 5'-3' $^{\prime}$ & Product size & Biotinylated \\
\hline 5'primer1 0367 & GGGGGTAATCCTCCCAAGTA & - & no \\
\hline 5'primer2 0367 & TCACCACACATCTCGTAGGC & - & no \\
\hline 5'primer3 0367 & TGCTGGGGGTCATAACTCTG & - & no \\
\hline N. punctiforme F-bio & TTACGCATCTCATCACGGGCCA & yes \\
\hline N. punctiforme R & ACAATACAAAAACACCTAGCCC & no \\
\hline N. 7120 F-bio & TGGCTATTAGTTGTATATTGT & yes bp \\
\hline N. 7120 R & TCGCTATTACGTTCCTCTCT & no \\
\hline EMSA PhupS F & TTCTAAAATTCTAGGGGAAATTG & no \\
\hline EMSA PhupS R & GGGCTAGGTGTTTTGTATTGT & no \\
\hline EMSA hupL F & CGCCATTATGAGGAAGCTGT & no \\
\hline EMSA hupL R & CGGTCTTCATCCAACCAATC & no
\end{tabular}

Sizes of the resulting PCR products are shown in base pairs (bp) 
determined using a Bio-Rad Protein Assay Dye Reagent (Bio-Rad Laboratories) according to manufacturer's protocol. For the DNA affinity assay a DNA fragment stretching from ATG (translation start point) for hupS to ATG for Npun_R0367 in N. punctiforme (from now on referred to as PhupS/Npun_R0367) and DNA fragment stretching ATG for hupS to ATG for asr0689 for Nostoc PCC 7120 (from now on referred to as PhupS/ asr0689), were amplified by PCR using the proofreading Phusion High Fidelity polymerase (Finnzymes) and the corresponding oligonucleotides (Figure 1, Table 3). The biotin labeled DNA fragments were incubated with streptavidin coated magnetic beads (Dynabeads ${ }^{\circledR}$ M-280, Dynal Biotech) and subsequently with protein extracts as previously described [32]. For each reaction $1.5 \mu \mathrm{g}$ DNA, $500 \mu \mathrm{g}$ beads and $1000 \mu \mathrm{g}$ proteins were used. Finally the beads were resuspended in $1 x$ SDS loading buffer containing $62.5 \mathrm{mM}$ Tris- $\mathrm{HCl} \mathrm{pH} \mathrm{6.8,} \mathrm{2 \%} \mathrm{SDS,}$ 2.5\% 2-mercaptoethanol, 7,5 mM Ditiotreitol (DTT), $10 \%$ glycerol and $5 \times 10^{-3} \%$ Bromphenol blue, and heated at $95^{\circ} \mathrm{C}$ for 5 minutes. The samples were excised from a $15 \%$ denaturing polyacrylamide gel, SDS-PAGE, stained with Brilliant Blue-G Colloidal (Sigma). Bands containing protein was cut from the gel and washed with water. The proteins were reduced by DTE and alkylated with iodoacetamide. After washing the proteins were digested with trypsin (Promega modified trypsin) in $50 \mathrm{mM} \mathrm{NH} 4 \mathrm{HCO} 3$ at $37^{\circ} \mathrm{C}$ over night. Peptides were extracted and the extract was subjected to MALDI-Tof analysis using a Bruker Ultraflex-Tof/Tof instrument. Proteins were identified by using the peptide maps for search in the NCBInr database using the Mascot search engine (http://MatrixScience.com). If needed for identification, MS/MS analysis was performed using the same MS instrument and search engine. The regions upstream of $16 S$ (Npun_r084 and rrn16Sc for N. punctiforme and Nostoc PCC 7120, respectively) were used as a negative control as well as beads incubated without DNA but with protein extract.

\section{Electrophoretic mobility shift assay}

For electrophoretic mobility shift assays, a fragment of the $N$. punctiforme hupS immediate upstream region was amplified by PCR using the proofreading Phusion High Fidelity polymerase (Finnzymes). The primers used were PhupS F and PhupS R yielding the 558 bp DNA fragment PhupS. Two control fragments, C1 and C2, were generated using primers against a region within the hupL gene, hupL F and hupL R. C1 was amplified with wild type $N$. punctiforme ATCC 29133 DNA as template, resulting in a $308 \mathrm{bp}$ fragment, and C2 was amplified using template DNA where the hupL gene has been interrupted by an antibiotic resistance cassette [33], resulting in a $1350 \mathrm{bp}$ fragment. Histidine-tagged
Nostoc PCC 7120 CalA was expressed in Nostoc PCC 7120 as described previously [34]. After expression, the protein was purified on a Superflow Ni-NTA column (Qiagen) according to the manufacturer's instructions. Binding of the protein to the DNA fragments was performed as described previously [35], using $100 \mathrm{ng}$ of each DNA fragment per $20 \mu$ reaction, and different amounts, from 0 to $45 \mathrm{ng}$, of purified His-tagged CalA. After incubation at room temperature for $1 \mathrm{~h}$, the assay mixtures were separated on an $8 \%$ non-denaturing polyacrylamide gel. The gel was subsequently stained with thiazole orange to visualize DNA bands.

\section{Results}

In silico genome analyses of the ORFs upstream of the hyp-genes demonstrates a conserved arrangement

To investigate if the same, or similar, conserved domains as reported for the five ORFs upstream of the hyp-genes in Nostoc PCC 7120 [19], asr0689 to alr0693, can be identified in the ORFs upstream of the hyp-genes in N. punctiforme, Npun_R0367 to Npun_R0363 (Figure $1)$, computational analyzes was performed. Indeed, in the deduced amino acid sequences of Npun_R0366 and Npun_0365, being the counterparts to asr0689 and asr0690 in Nostoc PCC 7120, putative transmembrane regions can be identified. Furthermore, in accordance to the findings in Nostoc PCC 7120 the deduced amino acid sequence of the alr0691 homologue Npun_R0367 and the alr0693 homologue Npun_0363 harbor TPR and NHL repeats, respectively. The deduced amino acid sequence of alr0692 was described as being similar to the C-terminal of NifU. When analyzing the homologue of alr0692 in N. punctiforme we found that Npun_R0364. as well was annotated as being similar to the C-terminal of NifU (Table 1).

\section{The ORFs upstream of the hyp-genes show the same pattern of transcription as the hyp genes}

To investigate if the ORFs upstream of the hyp-genes are expressed in the same manners as the hyp-genes and the structural genes of the hydrogenase and the nitrogenase, RNA was preparated from $N$. punctiforme and Nostoc PCC 7120 cultures 0, 24, 48 and 72 hours after nitrogen depletion, and from Nostoc PCC 7120 heterocysts 48 hours after nitrogen depletion. Note that the 0 hour sample represents a non $\mathrm{N}_{2}$-fixing condition. The control gene, 23S, showed, as expected, a constitutive high transcript level in both strains while the transcript levels of the structural genes nifD, hupS and the hyp-genes hypF and hypC were upregulated 24 hours after transition to $\mathrm{N}_{2}$-fixing conditions (Figures 2, 3). The five ORFs located upstream of the hyp-genes in both organisms showed similar transcript level patterns as nifD, hupS, hypF and hypC after nitrogen depletion. 


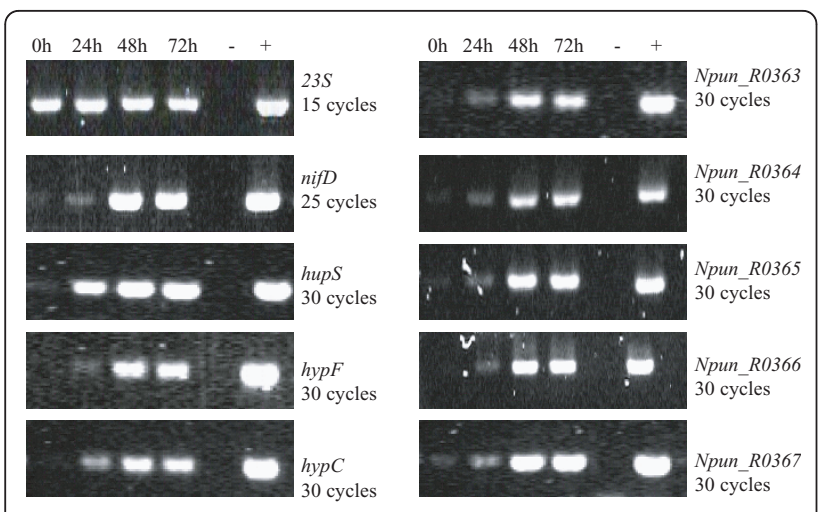

Figure 2 Transcript levels of the ORFs upstream of the hypgenes in Nostoc punctiforme ATCC 29133 after nitrogen depletion. Agarose gels showing the amplified PCR products using CDNA prepared from RNA from N. punctiforme cultures 0, 24, 48 and 72 hours after nitrogen depletion The tested genes are the hydrogenase, nitrogenase and ribosome structural genes hupS, nifD and 23S, the hyp-genes hypC and hypF and the ORFs upstream of the hyp-genes (Npun_R0363, Npun_R0364, Npun_R0365, Npun_R0366 and Npun_R03673). All DNA fragments were amplified with PCR using 30 cycles, except for nifD and 235 where 25 and 15 cycles were used, respectively. Negative (-) and positive controls (+) for the $P C R$ reactions are shown

The presence of transcript in RNA samples from heterocyst preparations showed that the expression takes place in the heterocyst and hence could be related to the expression of the uptake hydrogenase in Nostoc PCC 7120. In $N$. punctiforme the highest transcript levels for most genes occurred 48 hours after nitrogen depletion

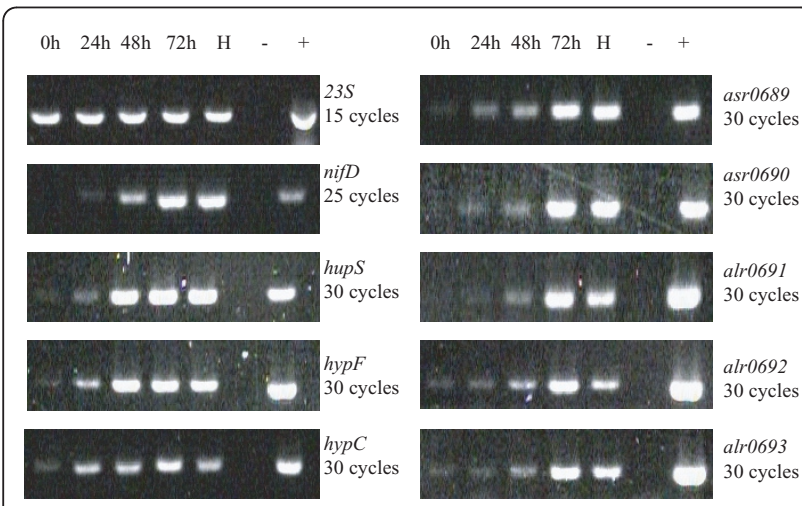

Figure 3 Transcript levels of the ORFs upstream of the hypgenes in Nostoc sp. strain PCC 7120 after nitrogen depletion. Agarose gels showing the amplified PCR products using CDNA prepared RNA from Nostoc PCC 7120 cultures 0, 24, 48 and 72 hours after nitrogen depletion as well as isolated heterocysts 48 hours after nitrogen depletion. The tested genes are the hydrogenase and ribosome structural genes hups and 23S, the hypgenes hypC and hypF and the ORFs upstream of the hyp-genes (asr0689, asr0690, alr0691, alr0691 and alr0693). All DNA fragments were amplified with PCR using 30 cycles, except for nifD and 235 where 25 and 15 cycles were used, respectively. Negative (-) and positive controls ( + ) for the PCR reactions are shown. while the highest transcript levels for most genes were found 72 hours after nitrogen depletion in Nostoc PCC 7120. The expression of $23 S$, used as a control representing a non-induced transcript, was constant for all time points in both strains. All the negative controls for the RT- reaction were blank (data not shown).

The genes upstream of the hyp-genes can be transcribed together with the hyp-genes as one operon

To investigate if the five ORFs upstream of the hypgenes, like in Nostoc PCC 7120, can form a transcript together with the hyp-genes regions between the genes were amplified from cDNA from $\mathrm{N}_{2}$-fixing cultures of $N$. punctiforme using gene specific primers for the individual genes. The results show that the region between hupS and Npun_R0367 can not be amplified. However, when using the gene specific primer Npun_R0364 for cDNA synthesis the intergenic regions between Npun_R0367-Npun_R0366, Npun_R0366-Npun_R0365, and Npun_R0365-Npun_R0364 can be amplified. In addition, the region between Npun_R0364-Npun_R0363 and Npun_R0363-hypF can be amplified using the gene specific primers Npun_R0363 and hypF respectively for the cDNA synthesis (Figure 4). A tsp could be identified 170 bp upstream of Npun_R0367. In the promoter region a putative -10 box was found (TATAGT) and possibly an imperfect -35 box (TAGAAT). The previously reported NtcA binding site [36] is centered -64.5 bp upstream of the tsp.

\section{CalA is binding to the hupSL Npun_R0367 promoter region in N. punctiforme and the hupSL asr6890 promoter region in Nostoc PCC 7120}

In order to identify potential transcriptional regulators interacting with the intergenic region harboring the hupS/Npun_R0367 promoter regions in N. punctiforme and the intergenic region harboring the hupS and the asr6890 promoter region in Nostoc PCC 7120, DNAprotein affinity assays were performed and proteins interacting with the promoter region fished out from a total protein extract of $\mathrm{N}_{2}$-fixing cultures. The resulting samples were run on a SDS-PAGE, bands were excised and the proteins analyzed with mass spectrometry. Among the identified peptides were CalA (encoded by Npun_R5944, alr0946) and CalB (encoded by Npun_R2896, all2080). The rest of the identified peptides, present in both negative controls and samples, were either from unspecific binding, e.g. phycobilisome linker polypeptide, or artifacts from the experimental procedure, e.g. streptavidin [see [32,37]]. The DNA affinity assay demonstrated an interaction of CalA and $\mathrm{CalB}$ to the intergenic region harboring the promoter region of the extended hyp-operon and the hupSL promoter region in $N$. punctiforme as well as in Nostoc 


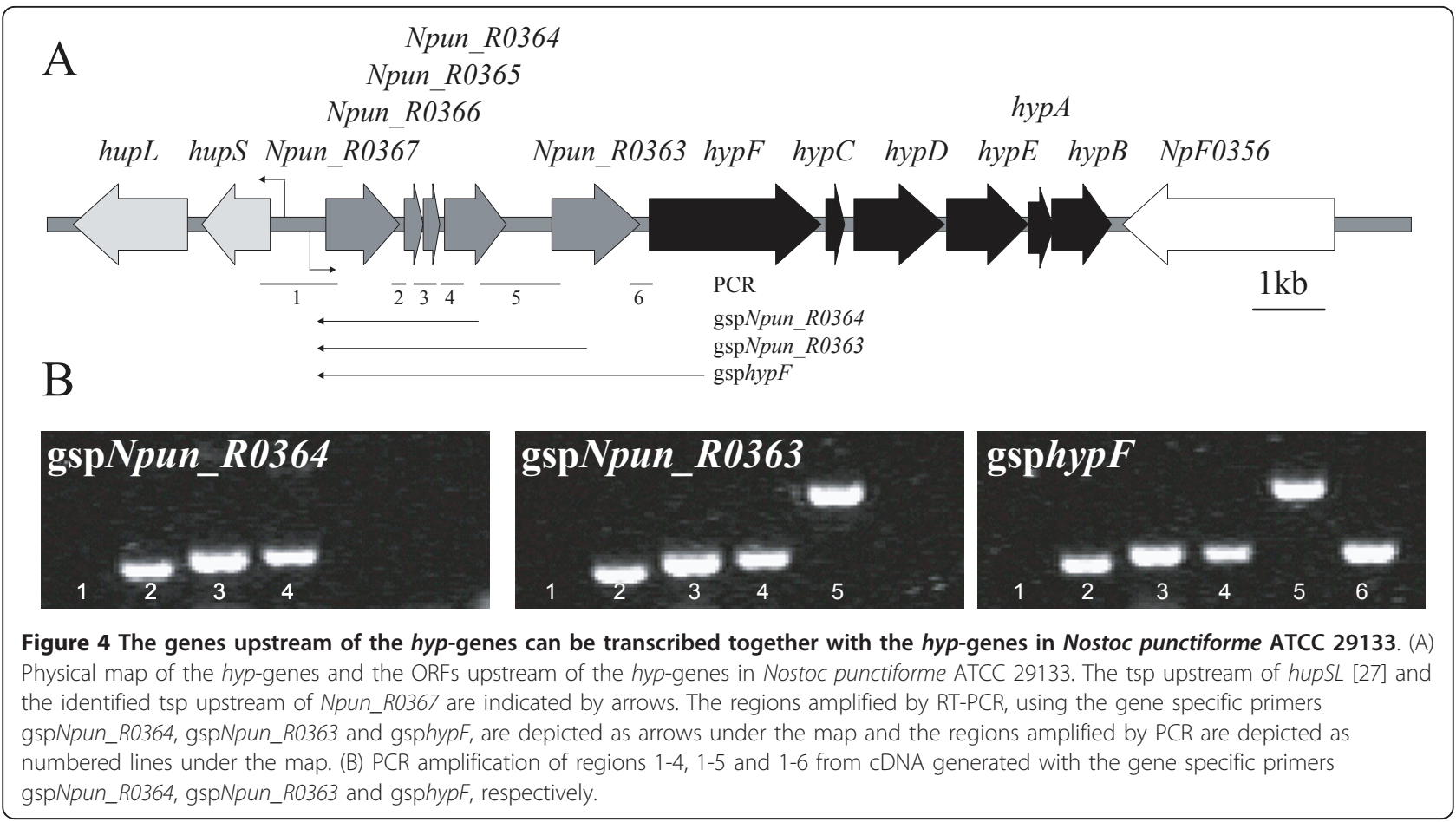

PCC 7120 (Figure 5). However, using this technique it is not possible to rule out that only one of the proteins is binding to the DNA and the second protein is interacting with the DNA binding protein. The expected sizes for CalA and CalB are approximately 16.2 and $15.3 \mathrm{kDa}$

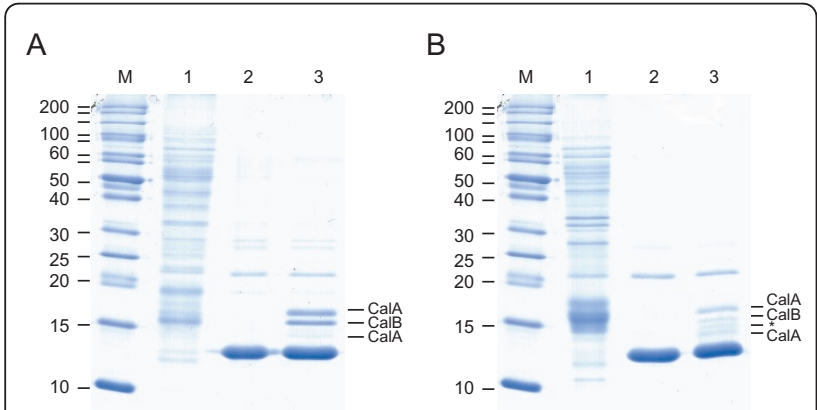

Figure 5 DNA affinity assay of the hupS/Npun_R0367 promoter region from Nostoc punctiforme ATCC 29133 and the hupS/ asr0389 promoter region from Nostoc sp. strain PCC 7120 and total protein extract from respective strain. SDS-PAGE of proteins interacting with (A) the hupS/Npun_R0367 promoter region from N. punctiforme and (B) the hupS/asr0389 promoter region from Nostoc PCC 7120 from DNA-protein affinity assays. Lanes: M) protein molecular weight marker; 1) Total protein extract, 2) DNA-free negative control, 3) hupS/Npun_R0367 or hupS/asr0389 promoter region respectively. The unlabelled bands on the gel, present in both negative controls and samples, correspond to identified peptides either from unspecific binding, e.g. phycobilisome linker polypeptide (weak bands), artifacts from the experimental procedure, e.g. streptavidin (strongest band) or peptides with too low concentration to be identified $\left(^{*}\right)$. in N. punctiforme and 16.1 and 15.3 in Nostoc PCC 7120 , respectively. Those sizes matches the sizes read from the gel with the exception of the lowest CalA band for Nostoc PCC 7120. Neither CalA nor CalB showed any interaction with the negative controls (16S data not shown). In order to confirm specific binding of CalA to the N. punctiforme hupS/Npun_R0367 promoter region, electrophoretic mobility shift assays were performed. A fragment of $N$. punctiforme DNA stretching from -1 to -558 bp upstream of the hupS start codon, was amplified by PCR and mixed with purified, histidine-tagged CalA from Nostoc PCC 7120 [34]. The CalA proteins from Nostoc PCC 7120 and N. punctiforme differ in only four positions of their predicted amino acid sequences (data not shown), and therefore, we expect that the purified Nostoc PCC 7120 CalA could substitute for $N$. punctiforme CalA in these experiments. To investigate non-specific binding of the protein to DNA, two control fragments, one a 308 bp part of the hupL gene, and the other the same part of hupL interrupted by an antibiotic resistance cassette, resulting in a 1350 bp fragment, were also included. When the reaction mixtures were separated by non-denaturing PAGE, the 558 bp DNA fragment from the hupS upstream region was retarded on the gel (Figure 6), while the two control fragments were not, demonstrating specific binding of CalA to the hupS upstream fragment. Electrophoretic mobility shift assays performed with the complete hupS/Npun_R0367 promoter region, $751 \mathrm{bp}$, showed the same specific binding of CalA (data not shown). 


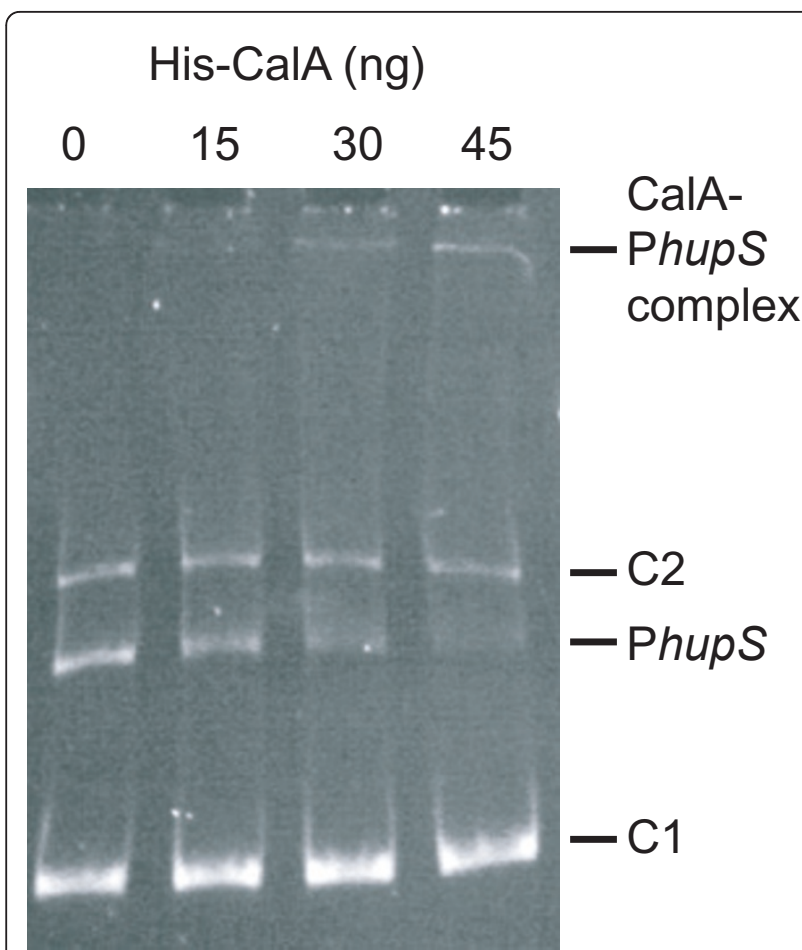

Figure 6 Electrophoretic mobility shift assay of the hupS/ Npun_R0367 promoter region in Nostoc punctiforme ATCC 29133. Electrophoretic mobility shift assay showing specific binding of purified CalA to the N. punctiforme hupS upstream region. C1 308 bp control fragment, C2 - 1350 bp control fragment, PhupS 558 bp hupS immediate upstream region fragment. $100 \mathrm{ng}$ of each fragment and increasing amounts (see label for each lane) of purified histidine-tagged CalA (His-CalA) from Nostoc PCC 7120 were used in the reaction mixtures.

\section{Discussion}

Here we show that the five ORFs upstream of the hypgenes in $N$. punctiforme harbors the same conserved regions as reported for Nostoc PCC 7120 [19]. The homologues alr0691 and Npun_R0367 contains TPR domains [19]. TPR domains mediate protein-protein interactions and are evolutionary conserved. They have been found in a variety of proteins involved in a wide range of cellular processes, for example protein folding. The numbers of TPR repeats differ between proteins, normally $3-16$, and they are not connected to any specific position in primary protein sequences [20-22]. Likewise the homologues alr0639 and Npun_R0363 harbor NHL repeats also believed to be involved in protein-protein interactions [23]. FeS clusters are important for electron transfer to and from the active site in hydrogenases. Assembly and insertion of FeS clusters is a complex process; Fe and $\mathrm{S}$ atoms must be mobilized from storages, transported to the correct cellular localization and inserted into the correct apoprotein. In the three FeS cluster assembly systems found so far the assistance of a scaffold protein and a cysteine desulfurase is needed $[3,9]$. Interestingly, homologue genes alr0692 and Npun_R0364 contain a conserved domain that is similar to the C-terminal domain of NifU. Proteins of the NifU family function as a scaffolds for FeS cluster assembly but another protein family, Nfu, with sequence similarity to the C-terminal domain of NifU has also been reported to function as biosynthetic scaffolds [3]. In cyanobacteria, Nfu have been proposed to be the primary FeS cluster scaffold protein [10].

The transcript level analysis of the ORFs upstream of the hyp-genes showed, as expected, an upregulation of transcripts for the control genes, nifD and hupS (nitrogenase and uptake hydrogenase structural genes) and $h y p F$ and hypC (hydrogenase maturation genes) when going from non $\mathrm{N}_{2}$ to $\mathrm{N}_{2}$-fixing conditions in both $N$. punctiforme and Nostoc PCC 7120. This increase in transcript levels was expected and has been shown before [6,27]. Similarly, an upregulation of the transcript levels for all five ORFs upstream of the hypgenes were seen after nitrogen depletion in both organisms while the amount of transcript of the control, $23 \mathrm{~S}$ (ribosomal subunit), were constant. For Npun_0363 this is in accordance with the earlier finding that this gene is expressed as one transcript with the hyp-genes [27]. Nostoc PCC 7120 harbors a bidirectional hydrogenase. To distinguish if an increase in transcript levels after a shift to $\mathrm{N}_{2}$-fixing conditions was connected to hydrogen metabolism in the heterocysts and not to bidirectional hydrogenase activity in the vegetative cells, heterocysts were isolated 48 hours after nitrogen depletion. The presence of transcripts of all genes tested in the heterocyst sample strongly indicates that expression takes place in the heterocysts. Since the ORFs upstream of the hyp-genes are not present in cyanobacterial strains containing only the bidirectional hydrogenase this points to a function related to the uptake hydrogenase. In Nostoc PCC 7120 the highest transcript levels of most genes investigated were detected 72 hours after nitrogen depletion while the highest transcript levels of most genes were detected already after 48 hours in $N$. punctiforme. The five ORFs upstream of the hyp-operon in N. punctiforme were shown to have, as hupGHIJK in R. leguminosarum [12], a tsp located upstream the gene cluster. In addition, as in Nostoc PCC 7120 [19], they are transcribed as a single operon and can form a transcript together with the hyp-genes in an extended hyp-operon. In bacterial genomes, genes that are functionally related are often clustered together and transcribed from the same promoter. By coordinating protein activities with gene regulation, genes expressed during different growth conditions, e.g. $\mathrm{N}_{2}$-fixation, can be turned on and off efficiently with minimal energy expenditure [38]. 
In Nostoc PCC 7120 a putative tsp was found upstream of asr0689 and in the promoter region a putative NtcA binding site and an extended -10 box were identified. In addition, tsps upstream of hypF and hypC were identified suggesting transcripts of varying sizes [19]. Occurrence of several tsps in one operon has been shown before in cyanobacteria and might allow for a more fine tuned regulation $[39,40]$. In $N$. punctiforme a tsp has been identified upstream of Npun_R0363 together with a putative -10 box and a putative NtcA binding site. No tsp has been found upstream of hypF or hypC [27]. In this study we found a novel tsp $170 \mathrm{bp}$ upstream of the translation start point, ATG, of Npun_R0367 as well as a - 10 and possibly a -35 box. NtcA is a global regulator of nitrogen metabolism in cyanobacteria $[41,42]$. The -10 box in the Npun_R0367 promoter match the consensus sequence $\left(\mathrm{TAN}_{3} \mathrm{~T}\right)$ reported for Class II NtcA activated promoters $[41,42]$, but there is no NtcA binding site overlapping the area of the -35 box. There is, however, a NtcA binding site located further upstream and we have earlier reported that NtcA does indeed interact specifically in EMSAs with this proposed binding site [43]. This NtcA binding motif was first associated transcription of hupSL and is centered at -258.5 in the hupSL promoter [36]. However, the promoter regions of hupSL and Npun_R0367 are located in the same intergenic region, although in opposite direction (Figure 1). In the Npun_R0367 promoter region the NtcA binding site is centered at -64.5 from the tsp. This shorter distance from the NtcA binding site to the tsp fits better, although not perfect, with the -41.5 distance reported for NtcA regulated promoters [41]. Interestingly, 10.5 base pairs approximately correspond to one helical turn of the DNA [23] which means that the NtcA site will have almost the reported angular orientation compared to the Npun_R0367 tsp. This, together with the importance of the NtcA binding site to the transcription of hupSL being low [43], supports our earlier suggestion that NtcA might not be involved in the regulation of hupSL but rather Npun_R0367, or putatively that NtcA is involved in the regulation of transcription from both promoters [43].

DNA binding assays showed that the novel transcriptional regulator CalA interacts specifically with the promoter region of the extended hyp-genes in both $N$. punctiforme and Nostoc PCC 7120 (Figure 5). Interestingly, two different bands were both identified as CalA. They may be either intact, cleaved, or partly degraded CalA, or different forms of CalA Furthermore, the CalA homologue, CalB, is also binding to the extended hyp promoter regions, or alternatively to CalA, in the respective strains (Figure 5). The cyanobacterial AbrB like Cal proteins belong to the transcription regulator family AbrB (Antibiotic resistance). They have a conserved DNA binding region in the C-terminal part of the protein, and the cyanobacterial $\mathrm{Cal}$ proteins may be classified into two separate clades, CalA and CalB $[34,44]$. AbrB is a global transition-state regulator well studied in Bacillus subtilis [44], that directly, or indirectly, regulates more than 60 different genes [45]. No consensus sequence for AbrB binding has been identified, instead the protein is believed to recognize a specific DNA 3D-conformation [46,47] and regulating by binding to promoter regions with different affinities [45] thereby fine-tuning the regulatory process. The functions of CalA and CalB in cyanobacteria are largely unknown, but parts of it are starting to be revealed. CalA appears to be essential, since no attempts to create a fully segregated knock-out mutant have been successful $[37,44]$, and have been shown to interact with the promoter regions of genes involved in hydrogen metabolism in Nostoc PCC 7120 [34] and Synechocystis PCC 6803 [37], toxin production in Aphanizomenon ovalisporum [48], and with the promoter regions of FtsZ [49] and FeSOD in Nostoc PCC 7120 [50]. Fully segregated knock-out mutants of CalB have, however, been constructed in Synechocystis PCC 6803 [44] and it appears to play an important role in the regulation of genes involved in nitrogen uptake [44] and carbon concentrating mechanisms [51]. Since CalA interacts with the promoter region of hypC in Nostoc PCC 7120 [34] it makes sense that CalA also is involved in the regulation of the upstream of the hyp-operon in N. punctiforme and Nostoc PCC 7120. Furthermore, due to the fact that the genes in the extended hyp-operon are expressed during nitrogen depletion, together with many other genes involved in $\mathrm{N}_{2}$-fixation and nitrogen metabolism, interaction of CalB with the extended hyp promoter region is not unreasonable. Whether CalA or CalB activates or represses transcription of the extended hyp-operon in N. punctiforme and Nostoc PCC 7120 remains to be revealed.

\section{Conclusions}

The five ORFs upstream of the hyp-genes in several filamentous $\mathrm{N}_{2}$-fixing cyanobacteria have an identical genomic localization, in between the uptake hydrogenase structural genes, hupSL, and the maturation protein genes, hypABCDEF. These ORFs are not present in strains harboring only the bidirectional hydrogenase. In N. punctiforme and Nostoc PCC 7120 they are transcribed as one operon and may form transcripts together with the hyp-genes. The homologues alr0691 and Npun_R0364 both contain a domain that is similar to NifU, a FeS cluster scaffold protein believed to be involved in the maturation of FeS cluster containing subunits. The expression pattern of the five ORFs within 
the extended hyp-operon in both Nostoc punctiforme and Nostoc PCC 7120 is similar to the expression patterns of hupS, nifD, hypF and hypC. CalA, a known transcription factor, interacts with the promoter region between hupSL and the five ORFs within the extended hyp-operon in both Nostoc strains.

\section{Acknowledgements}

This work was supported by the Swedish Energy Agency, the Knut and Alice Wallenberg Foundation, the Nordic Energy Research Program (project BioH2) and the European Union/Energy FP7 project SOLAR-H2 (contract \# 212508).

\section{Authors' contributions}

$\mathrm{MH}$ performed most of the experimental work and was involved in designing the experiments, analyzing the data and writing the manuscript. PiL carried out the electrophoretic mobility shift assays and was involved in writing of the manuscript. ÅA conceived the project and was involved in designing the experiments, and was involved in the transcriptional analysis. KS supervised the experimental work, participated in its design, and was involved in writing of the manuscript. PL coordinated the project and the writing of the manuscript. All authors have read and approved the manuscript.

\section{Author competing interests}

The authors declare that they have no competing interests.

Received: 7 February 2011 Accepted: 14 June 2011

Published: 14 June 2011

\section{References}

1. Ghirardi ML, Posewitz MC, Maness PC, Dubini A, Yu J, Seibert M: Hydrogenases and hydrogen photoproduction in oxygenic photosynthetic organisms. Annu Rev Plant Biol 2007, 58:71-91.

2. Tamagnini P, Leitao E, Oliveira P, Ferreira D, Pinto F, Harris DJ, Heidorn T, Lindblad P: Cyanobacterial hydrogenases: diversity, regulation and applications. FEMS Microbiol Rev 2007, 31:692-720.

3. Vignais PM, Billoud B: Occurrence, classification, and biological function of hydrogenases: an overview. Chem Rev 2007, 107:4206-4272.

4. Meeks JC, Elhai J: Regulation of cellular differentiation in filamentous cyanobacteria in free-living and plant-associated symbiotic growth states. Microbiol Mol Biol Rev 2002, 66:94-121.

5. Tsygankov AA: Nitrogen-fixing cyanobacteria: A review. Appl Biochem Microbiol 2007, 43:250-259.

6. Tamagnini P, Axelsson R, Lindberg P, Oxelfelt F, Wunschiers R, Lindblad P: Hydrogenases and hydrogen metabolism of cyanobacteria. Microbiol Mol Biol Rev 2002, 66:1-20.

7. Bock A, King PW, Blokesch M, Posewitz MC: Maturation of hydrogenases. Adv Microb Physiol 2006, 51:1-71.

8. Hoffmann D, Gutekunst K, Klissenbauer M, Schulz-Friedrich R, Appel J: Mutagenesis of hydrogenase accessory genes of Synechocystis sp. PCC 6803. Additional homologues of hypA and hypB are not active in hydrogenase maturation. FEBS J 2006, 273:4516-4527.

9. Fontecave M, Ollagnier-De-Choudens S: Iron-sulfur cluster biosynthesis in bacteria: Mechanisms of cluster assembly and transfer. Arch Biochem Biophys 2008, 474:226-237.

10. Balasubramanian R, Shen GZ, Bryant DA, Golbeck JH: Regulatory roles for IsCA and SufA in iron homeostasis and redox stress responses in the cyanobacterium Synechococcus sp strain PCC 7002. J Bacteriol 2006, 188:3182-3191.

11. Manyani H, Rey L, Palacios JM, Imperial J, Ruiz-Argueso T: Gene products of the hupGHIJ operon are involved in maturation of the iron-sulfur subunit of the [NiFe] hydrogenase from Rhizobium leguminosarum bv. viciae. J Bacteriol 2005, 187:7018-7026.

12. Martinez M, Brito B, Imperial J, Ruiz-Argueso T: Characterization of a new internal promoter (P3) for Rhizobium leguminosarum hydrogenase accessory genes hupGHIJ. Microbiol (Reading, England) 2004, 150:665-675.

13. Rey L, Hidalgo E, Palacios J, Ruiz-Argueso T: Nucleotide sequence and organization of an $\mathrm{H}_{2}$-uptake gene cluster from Rhizobium leguminosarum bv. viciae containing a rubredoxin-like gene and four additional open reading frames. J Mol Biol 1992, 228:998-1002.

14. Burgdorf T, Lenz O, Buhrke T, van der Linden E, Jones AK, Albracht SP, Friedrich B: [NiFe]-hydrogenases of Ralstonia eutropha $\mathrm{H} 16$ : modular enzymes for oxygen-tolerant biological hydrogen oxidation. J Mol Microbiol Biotechnol 2005, 10:181-196.

15. Menon AL, Mortenson LE, Robson RL: Nucleotide sequences and genetic analysis of hydrogen oxidation (hox) genes in Azotobacter vinelandii. J Bacteriol 1992, 174:4549-4557.

16. Vignais PM, Billoud B, Meyer J: Classification and phylogeny of hydrogenases. FEMS Microbiol Rev 2001, 25:455-501.

17. Bernhard M, Schwartz E, Rietdorf J, Friedrich B: The Alcaligenes eutrophus membrane-bound hydrogenase gene locus encodes functions involved in maturation and electron transport coupling. J Bacteriol 1996, 178:4522-4529.

18. Schubert T, Lenz O, Krause E, Volkmer R, Friedrich B: Chaperones specific for the membrane-bound [NiFe]-hydrogenase interact with the Tat signal peptide of the small subunit precursor in Ralstonia eutropha $\mathrm{H} 16$. Mol Microbiol 2007, 66:453-467.

19. Agervald A, Stensjö K, Holmqvist M, Lindblad P: Transcription of the extended hyp-operon in Nostoc sp. strain PCC 7120. BMC Microbiol 2008, 8:69.

20. Blatch GL, Lassle M: The tetratricopeptide repeat: a structural motif mediating protein-protein interactions. Bioessays 1999, 21:932-939.

21. D'Andrea LD, Regan L: TPR proteins: the versatile helix. Trends Biochem Sci 2003, 28:655-662.

22. Das AK, Cohen PW, Barford D: The structure of the tetratricopeptide repeats of protein phosphatase 5: implications for TPR-mediated protein-protein interactions. EMBO J 1998, 17:1192-1199.

23. Wagner R: The players or cellular components necessary for transcription. Transcriptional Regulation in Prokaryotes Oxford University Press, New York; 2000.

24. Carrasco CD, Buettner JA, Golden JW: Programed DNA rearrangement of a cyanobacterial hupL gene in heterocysts. Proc Natl Acad Sci USA 1995, 92:791-795.

25. Oxelfelt F, Tamagnini P, Lindblad P: Hydrogen uptake in Nostoc sp. strain PCC 73102. Cloning and characterization of a hupSL homologue. Arch Microbiol 1998, 169:267-274.

26. Gubili J, Borthakur D: Organization of the hupDEAB genes within the hydrogenase gene cluster of Anabaena sp. strain PCC7120. J Appl Phycol 1998, 10:163-167.

27. Hansel A, Axelsson R, Lindberg P, Troshina OY, Wunschiers R, Lindblad P: Cloning and characterisation of a hyp gene cluster in the filamentous cyanobacterium Nostoc sp. strain PCC 73102. FEMS Microbiol Lett 2001, 201:59-64.

28. Rippka R, Deruelles J, Waterbury JB, Herdman M, Stanier RY: Generic Assignments, Strain Histories and Properties of Pure Cultures of Cyanobacteria. J Gen Microbiol 1979, 111:1-61

29. Tamagnini P, Troshina O, Oxelfelt F, Salema R, Lindblad P: Hydrogenases in Nostoc sp. strain PCC 73102, a strain lacking a bidirectional enzyme. Appl Environ Microbiol 1997, 63:1801-1807.

30. Ow SY, Cardona T, Taton A, Magnuson A, Lindblad P, Stensjö K, Wright P. Quantitative shotgun proteomics of enriched heterocysts from Nostoc sp. PCC 7120 using 8-plex isobaric peptide tags. J Proteome Res 2008, 7:1615-1628

31. Ow SY, Noirel J, Cardona T, Taton A, Lindblad P, Stensjö K, Wright P: Quantitative overview of $\mathrm{N}_{2}$ fixation in Nostoc punctiforme ATCC 29133 through cellular enrichments and iTRAQ shotgun proteomics. J Proteome Res 2009, 8:187-198.

32. Oliveira P, Lindblad P: LexA, a transcription regulator binding in the promoter region of the bidirectional hydrogenase in the cyanobacterium Synechocystis sp PCC 6803. FEMS Microbiol Lett 2005, 251:59-66.

33. Lindberg P, Schütz K, Happe T, Lindblad P: A hydrogen-producing, hydrogenase-free mutant strain of Nostoc punctiforme ATCC 29133. Int J Hydrogen Energ 2002, 27:1291-1296.

34. Agervald A, Zhang X, Stensjö K, Devine E, Lindblad P: CalA, a cyanobacterial $A b r B$ protein, interacts with the upstream region of hypC and acts as a repressor of its transcription in the cyanobacterium Nostoc sp. strain PCC 7120. Appl Environ Microbiol 2010, 76:880-890. 
35. Montesinos ML, Muro-Pastor AM, Herrero A, Flores E: Ammonium/ methylammonium permeases of a cyanobacterium. Identification and analysis of three nitrogen-regulated amt genes in Synechocystis sp. PCC 6803. J Biol Chem 1998, 273:31463-31470.

36. Lindberg P, Hansel A, Lindblad P: hupS and hupL constitute a transcription unit in the cyanobacterium Nostoc sp PCC 73102. Arch Microbiol 2002, 174:129-133.

37. Oliveira P, Lindblad P: An AbrB-Like protein regulates the expression of the bidirectional hydrogenase in Synechocystis sp. strain PCC 6803. J Bacteriol 2008, 190:1011-1019.

38. Zheng Y, Szustakowski JD, Fortnow L, Roberts RJ, Kasif S: Computational identification of operons in microbial genomes. Genome Res 2002, 12:1221-1230.

39. Sjöholm J, Oliveira P, Lindblad P: Transcription and regulation of the bidirectional hydrogenase in the cyanobacterium Nostoc sp. strain PCC 7120. Appl Environ Microbiol 2007, 73:5435-5446.

40. Valladares A, Muro-Pastor AM, Herrero A, Flores E: The NtcA-dependent P1 promoter is utilized for $g \ln A$ expression in $\mathrm{N}_{2}$-fixing heterocysts of Anabaena sp. strain PCC 7120. J Bacteriol 2004, 186:7337-7343.

41. Herrero A, Muro-Pastor AM, Flores E: Nitrogen control in cyanobacteria. J Bacteriol 2001, 183:411-425.

42. Luque I, Flores E, Herrero A: Molecular Mechanism for the Operation of Nitrogen Control in Cyanobacteria. EMBO J 1994, 13:2862-2869.

43. Holmqvist M, Stensjö K, Oliveira P, Lindberg P, Lindblad P: Characterization of the hupSL promoter activity in Nostoc punctiforme ATCC 29133. BMC Microbiol 2009, 9:54.

44. Ishii A, Hihara Y: An AbrB-like transcriptional regulator, SII0822, is essential for the activation of nitrogen-regulated genes in Synechocystis sp PCC 6803. Plant Physiol 2008, 148:660-670.

45. Bobay BG, Benson L, Naylor S, Feeney B, Clark AC, Goshe MB, Strauch R, Thompson R, Cavanagh J: Evaluation of the DNA binding tendencies of the transition state regulator AbrB. Biochemistry 2004, 43:16106-16118.

46. Strauch MA, Spiegelman GB, Perego M, Johnson WC, Burbulys D, Hoch JA: The transition state transcription regulator $A b r B$ of Bacillus subtilis is a DNA binding protein. EMBO J 1989, 8:1615-1621.

47. Xu K, Strauch MA: In vitro selection of optimal AbrB-binding sites: comparison to known in vivo sites indicates flexibility in AbrB binding and recognition of three-dimensional DNA structures. Mol Microbiol 1996, 19:145-158.

48. Shalev-Malul G, Lieman-Hurwitz J, Viner-Mozzini Y, Sukenik A, Gaathon A, Lebendiker M, Kaplan A: An AbrB-like protein might be involved in the regulation of cylindrospermopsin production by Aphanizomenon ovalisporum. Environ Microbiol 2008, 10:988-999.

49. He $D, X u X$ : CalA, a cyAbrB protein, binds to the upstream region of $f t s Z$ and is down-regulated in heterocysts in Anabaena sp. PCC 7120. Arch Microbiol 2010, 192:461-469.

50. Agervald A, Baebprasert W, Zhang X, Incharoensakdi A, Lindblad P, Stensjö K: The CyAbrB transcription factor CalA regulates the iron superoxide dismutase in Nostoc sp. strain PCC 7120. Environ Microbiol 2010, 12:2826-2837.

51. Lieman-Hurwitz J, Haimovich M, Shalev-Malul G, Ishii A, Hihara $Y$, Gaathon A, Lebendiker M, Kaplan A: A cyanobacterial AbrB-like protein affects the apparent photosynthetic affinity for $\mathrm{CO}_{2}$ by modulating low$\mathrm{CO}_{2}$-induced gene expression. Environ Microbiol 2009, 11:927-936.

\section{Submit your next manuscript to BioMed Central and take full advantage of:}

- Convenient online submission

- Thorough peer review

- No space constraints or color figure charges

- Immediate publication on acceptance

- Inclusion in PubMed, CAS, Scopus and Google Scholar

- Research which is freely available for redistribution

Submit your manuscript at www.biomedcentral.com/submit
C Biomed Central 